\title{
Analytical procedure for geometrical evaluation of flat surfaces formed by multiple indentation processes
}

\author{
C. Bernal ${ }^{1, a}$, A.M. Camacho ${ }^{1, b}$, J.M. Arenas ${ }^{2, c}$, E.M. Rubio ${ }^{1, d}$ \\ ${ }^{1}$ Department of Manufacturing Engineering, National Distance University of Spain (UNED), Madrid, \\ Spain \\ ${ }^{2}$ Department of Design and Product Engineering, Technical University of Madrid, Madrid, Spain \\ acbernal@ind.uned.es, bamcamacho@ind.uned.es, cjosemanuel.arenas@upm.es, \\ derubio@ind.uned.es
}

Keywords: Forming process, indentation, geometric surface, effective surface, reverse engineering, punch depth, shape factor.

\begin{abstract}
Elementary indentation processes are relatively simple compression processes and they are quite extended in many fields of the industry. Nevertheless, the concatenation of these processes is a new approach and has interest from a technological point of view. In this work the geometry of multiple indentation processes is analyzed on flat surfaces. The state of the formed surface by several types of tools is studied and a model has been developed in order to quantify by means of shape parameters the quality of the theoretical surface depending on the technological and geometrical characteristics of the process.
\end{abstract}

\section{Introduction}

In conventional indentation operations a force is applied on a workpiece by means of an indenter or punch in order to create an impression on the surface of the part. Indentation is a compression process that generates permanent plastic deformations on the workpiece and so it can be easily assimilated to forging processes. However, it is considered a "secondary process" because the deformations obtained are small, very localized and they have typically a superficial effect [1].

Nevertheless, the physical fundamentals of indentation processes are closely related to some other technological tests and processes such as the classical hardness tests (for example, Brinell, Rockwell, Vickers), coining processes and/or shot peening. Obviously, the main aim in each of them differs from one to another, but in all the cases the geometrical component is a relevant factor to consider additionally to the metal-mechanic analysis of processes.

A recent proposal of innovative process, the Localized-Incremental Forging Process (LIFP) [2], is focused on obtaining the final part as the result of discrete indentations by a simple tool of general purpose and small size [3]. This way, the final geometry of the workpiece is achieved by means of the displacement of the tool throughout the volume of work. Some of the main advantages of LIFP are that applied forces are lower than in conventional forging processes, complex dies are not required (a simple punch can be used instead of them) and the process can be developed in a CNC machine, increasing the flexibility of the process [2].

In this new forming strategy, besides the unitary forming stages, it is important to evaluate the global geometrical result that comes from the application of multiple strokes as it is done in other incremental forming processes $[4,5]$; being the expected surface a function of the punch geometry but also of some other process parameters such as the step between localized operations and the punch depth.

In this work the geometrical response of these multiple indentation operations is studied as a function of the formerly mentioned technological-geometrical parameters (punch geometry, step and punch depth), considering the most common punch geometries: spherical punch and flat punch with toric fillet.

Subsequently, a specific method is developed in order to assess the surface texture after the application of this kind of process, and a particular methodology is proposed to quantify somehow the surface characteristics of the geometries to obtain and the efficiency of the trajectories to be applied. 


\section{Geometric definition of the process}

In a multiple indentation process, the forming action is sequentially repeated following a defined trajectory or path. The final result of the formed part typically depends on the tool geometry, its progressive positioning defined by the steps along two directions, $a$ and $b$, and the punch depth, $C$.

Below, the multiple indentation process is analyzed from a geometric point of view to predict the expected geometric surface, even though other elements have to be also considered, such as the volume constancy principle. In this case, it is recommended to analyze the actual formed workpiece (the effective surface). The results of this evaluation can be compared with the theoretical analysis that is approached in this paper, considering it like a reference guide.

Different formed surface textures can be obtained with tools of different typology. From a theoretical point of view, a multiple indentation with a cylindrical flat punch does not create any impression on the surface. However, it is interesting the study with spherical punches and flat punches with toric fillet. These cases are the most common situations to deal with, having special interest the study of geometries obtained by indentations with overlapping of the tool and also of the impression (trace).

In order to evaluate the geometrical characteristics and the surface textures some parameters related to the tool and the process are defined:

$R:$ radius of the tool

$R_{p}:$ radius of the spherical end of the tool

$r$ : radius of the torical end of the tool $a$ : step along one direction

$b:$ step along the orthogonal direction

$C$ : tool depth

Spherical punch. It is possible to study at the same time two cases: when the tool ends in a spherical cap, by assuming $R_{p}>R$, and when it is a hemispherical punch, that is $R_{p}=R$. In Fig. 1 the impressions generated by a hemispherical punch are shown; this multiple indentation process comprises four strokes, there is overlapping of the tool and the orthogonal steps, $a$ and $b$, can also be identified. Fig. 2 is an example of actual part composed of eight strokes with hemispherical impressions overlapped that has been digitized by a 3D scanning technique.

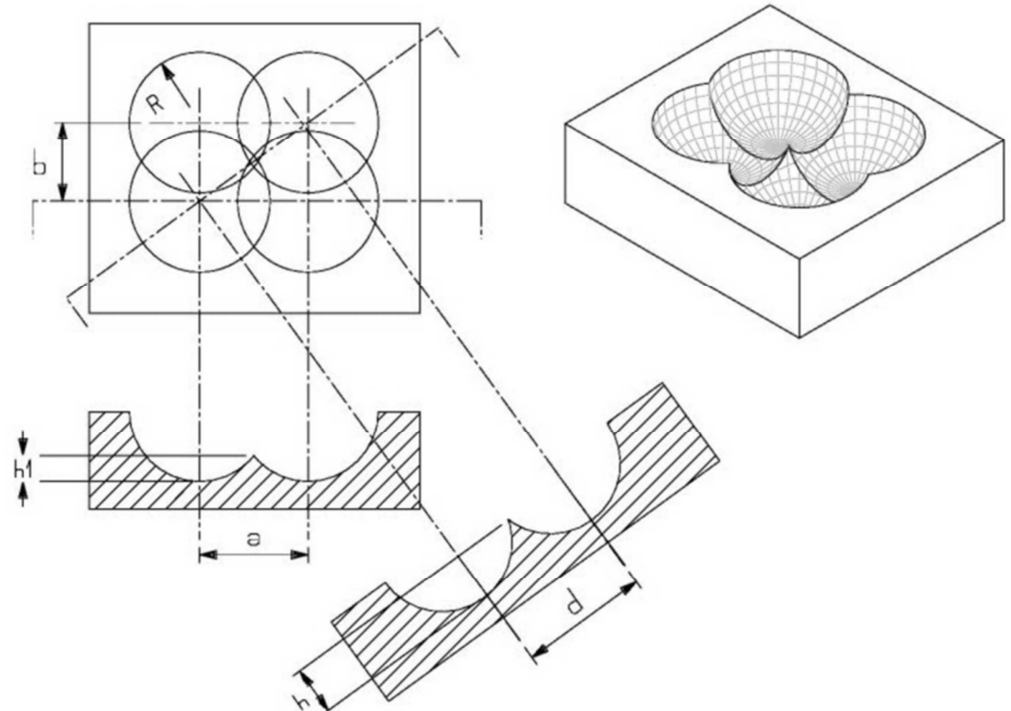

Fig. 1. Multiple indentation with hemispherical punch.

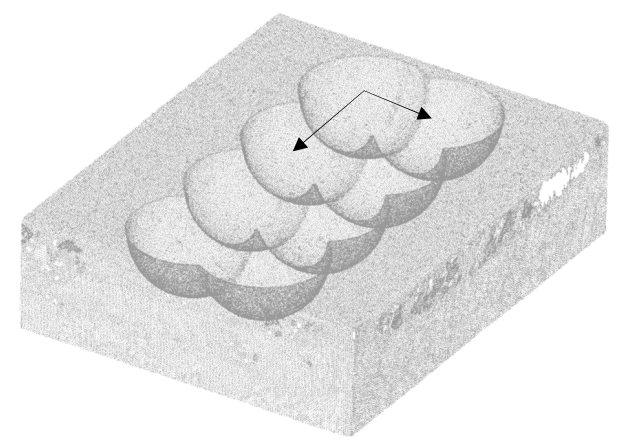

Fig. 2. Multiple indentation with hemispherical punch digitized by 3D scanning (Comet L3D).

The planar cuts show the direction of higher irregularities on the surface, being $d$ the equivalent step on the less favourable plane. 
Flat punch with toric fillet. In this case the torical radius, $r$, is introduced. Again, the impression depends on the orthogonal steps $a$ and $b$, on the radii $R$ y $r$ and on the punch depth, $C$ (Fig. 3).
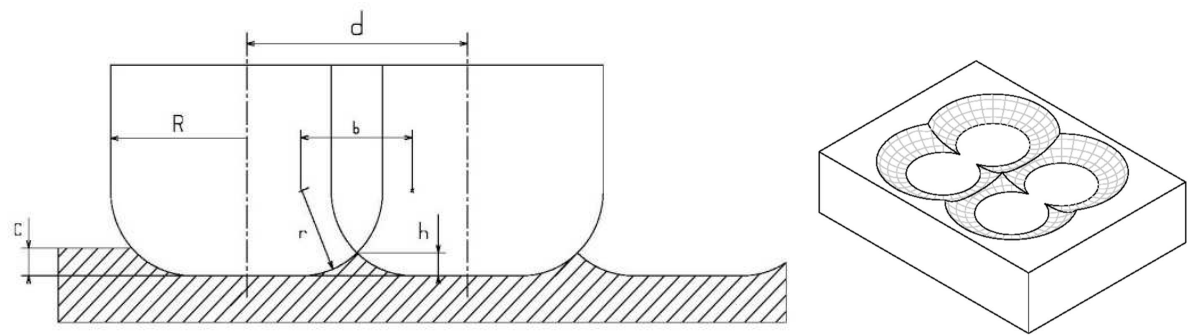

Fig. 3. Multiple indentation with flat punch with toric fillet.

Overlapping of the impression. When typifying the different cases, both the overlapping of the impression and the overlapping of the tool have been mentioned. The analysis of the different impressions generated by several types of punches shows that, depending on the values of tool dimensions, steps and tool depth, overlapping of the tool can exist but not on the impression. The evaluation model of "expected surface" proposed below determines the required conditions to obtain overlapping on the impression.

\section{Evaluation model of expected surface}

\section{Shape factors of the surface}

Spherical punch. Objective criteria can be established to assess the surface texture of the formed part for the cases formerly described. At first, the most generic case (that is, the punch with spherical cap) is analyzed. Fig. 4 represents an indentation process with overlapping of the impression by means of a tool with spherical cap end.

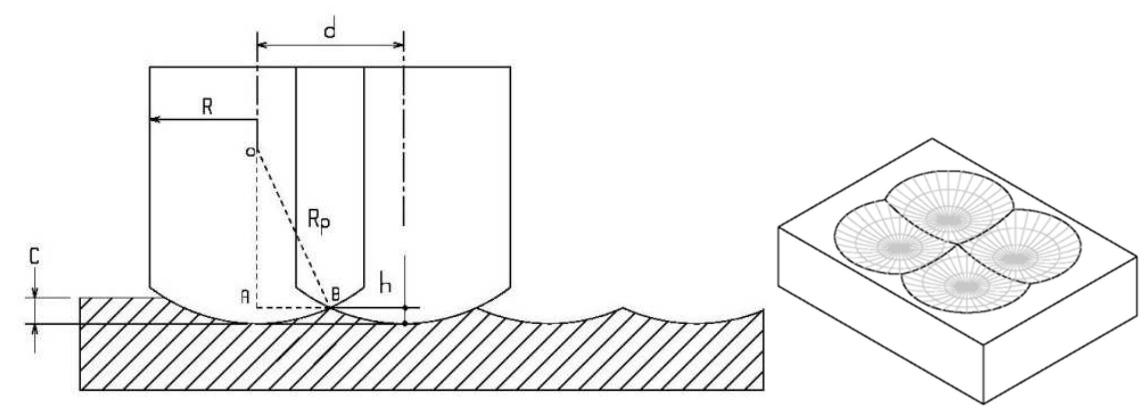

Fig. 4. Multiple indentation process with overlapping of the impression by means of a tool with spherical cap end.

The proposed model is based on the standardized evaluation of the surface roughness, as it is defined by international standards [6, 7]. Thus, two shape evaluation parameters can be identified, named shape factors and denoted by $S_{M}$ and $S_{a}$. These factors quantify the maximum deviations and the mean values that can be obtained on the basis of the expected surface. The shape factors $S_{M}$ and $S_{a}$ highly depend on the tool characteristics and on the process parameters.

$S_{M}$ represents the maximum irregularity of the surface and $S_{a}$ is the mean irregularity along the distance $l$, and both can be expressed by the following equations:

$$
S_{M}=h ; \quad S_{a}=\frac{1}{l} \int_{0}^{l}|z| \cdot d x
$$


Calculation of $\boldsymbol{S}_{\boldsymbol{M}}$. The shape factor $S_{M}$ quantifies the height of the peak of the maximum impression $(h)$ for a multiple indentation with steps $a$ and $b$.

Considering the triangle OAB of Fig. 4 , and when there is overlapping $(d<2 R)$, the value of $S_{M}$ can be determined as follows:

$$
S_{M}=R_{p}-\sqrt{R_{p}{ }^{2}-\left(\frac{a^{2}+b^{2}}{4}\right)} .
$$

Whereas when there is not overlapping $(d>2 R)$ :

$$
S_{M}=\mathrm{C}
$$

From the former equations it can be stated that if there is no overlapping of the tool, $S_{M}$ only depends on the punch depth.

On the contrary, with overlapping of the tool, $S_{M}$ depends on the steps $a$ and $b$ and on the radius of the spherical part of the tool, getting more smoothed surfaces when $R_{p}>>d$.

On the other hand, the punch penetration required to get overlapping on the impression is reached when $C=S_{M}$, being limited the value of the punch depth $C$ (with overlapping on the impression) by means of the following expression:

$$
C \geq R_{p}-\sqrt{R_{p}{ }^{2}-\left(\frac{a^{2}+b^{2}}{4}\right)} .
$$

Calculation of $\boldsymbol{S}_{\boldsymbol{a}}$. The shape factor $S_{a}$ allows to predict the mean deviation or irregularity on the surface (Eq. 1).

In Fig. 5 a graphical scheme is presented in order to explain the procedure for the calculation of $y_{c}$ (distance of the central line that equals the areas above and below) and the shape factor $S_{a}$.

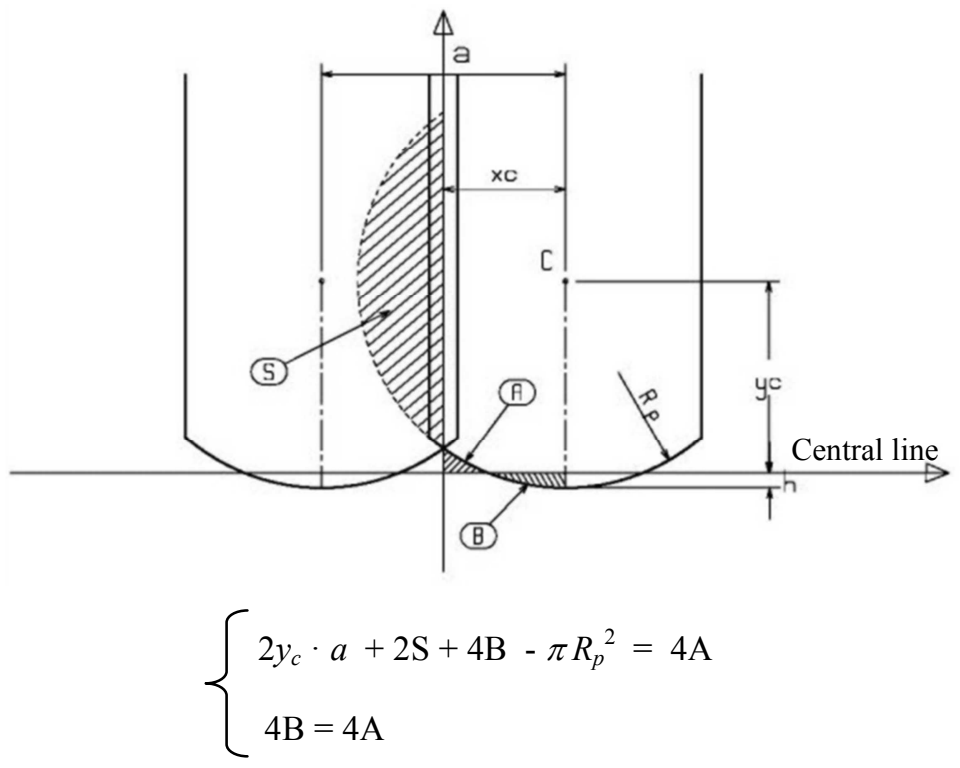

Fig. 5. Scheme for the calculation of $y_{c}$ and the shape factor $S_{a}$.

The circular segments $S$ and $B$ are calculated by knowing the location of the cut segment [7], being finally the expressions of $y_{c}$ and $S_{a}$ as follows: 


$$
\begin{aligned}
& y_{c}=\frac{\pi R_{p}{ }^{2}-2 S}{2 a}=\frac{\pi R_{p}{ }^{2}-2\left[R_{p}{ }^{2} \cos ^{-1}\left(\frac{a}{2 R_{p}}\right)-\frac{a}{2} \sqrt{R_{p}{ }^{2}-\left(\frac{a}{2}\right)^{2}}\right]}{2 a} . \\
& S_{a}=\frac{4 B}{a}=\frac{2\left[R_{p}{ }^{2} \cos ^{-1}\left(\frac{y_{c}}{R_{p}}\right)-y_{c} \sqrt{R_{p}{ }^{2}-y_{c}{ }^{2}}\right]}{a} .
\end{aligned}
$$

In the case of hemispherical punch, shape factors $S_{M}$ and $S_{a}$ and the minimum penetration $C$ to obtain an overlapping of the impression follow the same formulation given in Eq. 2, 3, 4 and 6, considering in this case $R_{p}=R$.

Flat punch with toric fillet. The shape factor $S_{M}$ is calculated in a similar way for a partial overlapping of the impression, that is, when $2(R-r)<d<2 R$ :

$$
S_{M}=r-\sqrt{r^{2}-\left[\frac{\sqrt{a^{2}+b^{2}}}{2}-(R-r)\right]^{2}} .
$$

For a complete overlapping of the impression, i.e. when $d<2(R-r)$, irregularities on the surface are not generated. In this case, the penetration value to obtain overlapping of the impression is defined by Eq. 8.

$$
C \geq r-\sqrt{r^{2}-\left[\frac{\sqrt{a^{2}+b^{2}}}{2}-(R-r)\right]^{2}} .
$$

$S_{a}$ is calculated following the same methodology than in the former case, overlapping locally the toric radius in the same way and solving the problem by means of an iterative procedure.

In Table 1 results for three types of punches and different steps values are presented.

Table 1. Comparative table of the shape factors for three different types of punches and different steps values.

\begin{tabular}{|c|c|c|c|c|c|c|c|c|c|c|c|c|c|c|c|}
\cline { 2 - 14 } \multicolumn{1}{c|}{} & \multicolumn{4}{c|}{ Hemispherical, $R=10$} & \multicolumn{4}{c|}{ Spherical, $R=10, R_{p}=50$} & \multicolumn{5}{c|}{ Torical, $R=10, r=3$} \\
\hline \multirow{3}{*}{$a$} & \multicolumn{4}{|c|}{$0<a<2 R$} & \multicolumn{4}{c|}{$0<a<2 R$} & \multicolumn{4}{c|}{$2(R-r) 2 R$} \\
\cline { 2 - 16 } & 0 & 5 & 10 & 15 & 20 & 0 & 5 & 10 & 15 & 20 & 14 & 15.5 & 17 & 18.5 & 20 \\
\hline$S_{M}$ & 0 & 0.317 & 1.339 & 3.386 & 10 & 0 & 0.062 & 0.250 & 0.566 & 1.010 & 0 & 0.095 & 0.402 & 1.02 & 3 \\
\hline$S_{a}$ & 0 & 0.081 & 0.338 & 0.828 & 1.81 & 0 & 0.016 & 0.064 & 0.144 & 0.258 & 0 & 0.005 & 0.039 & 0.122 & 0.297 \\
\hline
\end{tabular}

Values of $S_{M}$ and $S_{a}$ gathered in Table 1 have been calculated in the case of overlapping of the impression. The cases where there is no overlapping have been not considered because they are less interesting from the point of view of the surface texture evaluation. The shape factors have been obtained for different values of step $a$ (being $a=b$ in this case); this way the step range goes from 0 to the tangential position $(a=2 R)$. For the punch with toric fillet, the lower step value is defined as $2(R$ $-r)$, not existing impression with lower steps.

The shape factors $S_{M}$ and $S_{a}$ allow to quantify and to bound the values of maximum and mean irregularities of a formed surface, taking into account not only the geometrical but also the technological characteristics of the process. 
On the other hand, it is possible to analyse the surface texture generated experimentally through triangular STL mesh of digitized effective surface. In this case, the evaluation of the volume under the scanned surface allows positioning of the central plane and calculation of shape factors. The position of central plane is given by the quotient of the volume under the scanned surface and the area of the projected scanned surface.

\section{Conclusions}

Multiple indentation processes have been studied from a geometrical point of view, analysing the factors that influence the surface textures obtained. It is observed that the sequenced application of localized indentation processes generates a "surface effect" on the workpieces to be formed. These processes have particular geometrical limitations depending on the geometric-technological parameters, and the study presented in this paper allows predicting the best surface obtainable or expected surface. Indentation processes of flat surfaces by means of spherical tools and flat tools with toric fillet have been analysed. Geometrical analysis provides the methodological approach necessary to evaluate the surface textures generated experimentally in actual multiple indentation processes. Using this approach and reverse engineering of the effective surface it is possible to state more complex cases, whit no flat surfaces and different typologies of tool, in order to predict the quality of the surfaces obtained.

\section{Acknowledgements}

This work was financially supported by the Ministry of Economy and Competitiveness of Spain (Project DPI2009-07300) and the funds provided trough the Annual Grant Call of the E.T.S. Ingenieros Industriales (Industrial Engineering School) of UNED.

\section{References}

[1] J. Chakrabarty: Theory of Plasticity (Elsevier Butterworth-Heinemann, Oxford, 2006).

[2] A.M. Camacho, M.M. Marín, C. Bernal and M.A. Sebastián, in: Proceedings of the European Simulation and Modelling Conference 2011 (ESM 2011), edited by EUROSIS/Ghent University, Ghent, Guimaraes (2011).

[3] A.M. Camacho, M.M. Marin, E.M. Rubio and M.A. Sebastián: Mater. Sci. Forum Vol. 713 (2012), p. 19.

[4] F. Micari, G. Ambrogio and L. Filice: J. Mater. Process. Tech. Vol. 191 (2007), p. 390.

[5] C.J. Luis, J. Vivancos and M.A. Sebastián: Precis. Eng. Vol. 25 (2001), p. 1.

[6] ISO 4287:1997. Geometrical Product Specifications (GPS). Surface texture: Profile method. Terms, definitions and surface texture parameters. International Organization for Standardization (ISO), Geneva, 1997.

[7] ISO 4288:1996. Geometrical Product Specifications (GPS). Surface texture: Profile method. Rules and procedures for the evaluation of surface texture. International Organization for Standardization (ISO), Geneva, 1996. 